Inventory Management of Remanufacturable Products

Toktay, Wein and Zenios

\#3965-97-MSA

June, 1997 


\title{
Inventory Management of Remanufacturable Products
}

\author{
L. Beril Toktay \\ Operations Research Center, M.I.T. \\ Cambridge, MA 02139 \\ Lawrence $M$. Wein \\ Sloan School of Management, M.I.T. \\ Cambridge, MA 02139 \\ Stefanos A. Zenios \\ Graduate School of Business, Stanford University \\ Stanford, CA 94305
}

\begin{abstract}
We address the procurement of new components for recyclable products in the context of Kodak's single-use camera. The objective is to find an ordering policy that minimizes the total expected procurement, inventory holding and lost sales cost. Distinguishing characteristics of the system are the uncertainty and unobservability associated with return flows of used cameras. We model the system as a closed queueing network, develop a heuristic procedure for adaptive estimation and control, and illustrate our methods with disguised data from Kodak. Using this framework, we investigate the effects of various system characteristics such as informational structure, procurement delay, demand rate and length of the product's life cycle.
\end{abstract}

June 1997 


\section{Introduction}

Remanufacturing is the process by which used products are recovered, processed and sold as new products. It introduces a host of issues related to product design, production planning, inventory control, logistics, information systems, marketing and quality control, as discussed in Thierry et al. (1995). Remanufacturing complicates inventory management by introducing return flows of used products, which are uncertain with regards to their quantity, timing and quality.

This paper is motivated by the supply chain for Kodak's single-use flash camera. This chain includes the procurement of circuit boards from the supplier, the production or remanufacture of cameras at the Kodak facility, and their distribution, sales and recovery. Each flash camera includes one circuit board, which is the primary cost driver for this product. The circuit boards are manufactured overseas and shipped to Kodak's production facility in Rochester, NY. The shipping delay is substantial and necessitates large circuit board inventories. The production facility operates as a flow line, using both old and new components to manufacture new cameras. Completed cameras are then transported through Kodak's distribution system to various retail outlets. About $75 \%$ of sales are impulse buys (e.g., bought while on vacation, Goldstein 1994), so that most unsatisfied demand is lost. Customers take the used cameras to a photofinishing laboratory, where the film is taken out and processed. The laboratories receive a rebate for each used camera they subsequently return to Kodak. Despite this, Kodak receives only a portion of the cameras sold. The reusable parts (the circuit board, plastic body and lens aperture) of the returned cameras are put back into production after inspection.

When our project began, Kodak managers did not have a precise estimate of what portion of sold cameras are returned, and had not attempted to estimate how long it takes for a camera to return to Kodak. Even if these estimates were known, the inventory management problem is complicated by the fact that the portion of the supply chain from a sale to a return cannot be observed by the manufacturer. A literature survey on production planning and inventory control in the context of remanufac- 
turing (van der Laan, Salomon and Dekker 1995) reveals that these key aspects of remanufacturing systems have been, for the most part, overlooked by researchers. An exception is Kelle and Silver (1989a,b), who model returns as a function of past sales (via a known delay distribution) and estimate return flows from past sales and returns. Otherwise, returns are assumed to be independent of past sales, and most authors assume that the sales and return processes are independent. Regardless of specific assumptions about the return flows, all existing models assume that the statistical characteristics of these flows or of their relationship to the sales process are known (e.g., returns are Poisson with known rate).

We allow returns to depend on past sales, and assume that the parameters underlying the statistical models describing this relationship are unknown. Then we use evolving sales and returns data to dynamically estimate these statistical characteristics and the return flows, and incorporate this information into our inventory management decisions. More specifically, we construct a queueing network in $\S 2$ to model the entire supply chain, which consists of procurement, shipping, production, distribution, sales and returns. The process between sales and returns constitutes the unobservable portion of this network. To model the return flows, we let returns depend on sales through an unknown return probability and delay distribution. Our objective is to develop a simple and effective procurement policy for new circuit boards, which are the most costly component. To this end, we consider a class of aggregate base stock policies, which controls the total number of circuit boards in the supply chain (including the unobservable portion). This type of policy is similar in spirit to the CONWIP policy (e.g., Solberg 1977, Whitt 1984, Spearman et al. 1990), but is applied at the supply chain level rather than the factory floor level (Rubio and Wein 1996). In $\S 3.1$ we use Bayesian statistics and survival analysis to dynamically estimate probability distributions for the return probability and the return delay based on past sales and returns. In $\S 3.2$ we use methods developed by Kelle and Silver (1989a) to dynamically estimate the total number of circuit boards in the system as a function of the estimated return flow parameters and historical information about sales and returns. This analysis is combined with the fixed population mean method 
(Whitt 1984) to construct a heuristic dynamic aggregate base stock policy.

In $\S 4$ we use computer simulation to investigate the cost impact of different informational structures and of the way in which available information is utilized, and how these effects vary with demand rate, procurement delay and the length of the product's life cycle. Concluding remarks are given in $\S 5$.

\section{The Model}

We model the supply chain as a queueing network in $\S 2.1$, and define the class of circuit board procurement policies in $\S 2.2$.

\subsection{The Queueing Network}

The queueing network has six nodes and is depicted in Figure 1. The performance of the system depends on the service time distributions only through their means, since our queueing network is of product form (Baskett et al. 1975, Kelly 1979). We model the vendor node as a single-server queue because Kodak's circuit boards comprise most of the output at the circuit board supplier's production facility. Service times at this node are exponential with rate $\mu_{v}$. Orders placed for circuit boards constitute the arrivals to this node.

Completed circuit boards enter the shipping node, which is an infinite-server node with general service times. The mean service time, or shipping delay, is $1 / \mu_{s}$, and all departures from this node go to the production node. We assume that whenever new or used circuit boards are available at the production facility, cameras will be manufactured; i.e., all other components required for camera production are readily available. The production facility is modeled as a single-server queue with exponential service times with rate $\mu_{p}$.

All manufactured cameras are transported to retailers via a vast distribution system. The time the cameras spend in the distribution node is modeled as an infiniteserver queue, and the delay is generally distributed with mean $1 / \mu_{d}$. All departures go to the retailer node, where the cameras reside until they are purchased. We model 


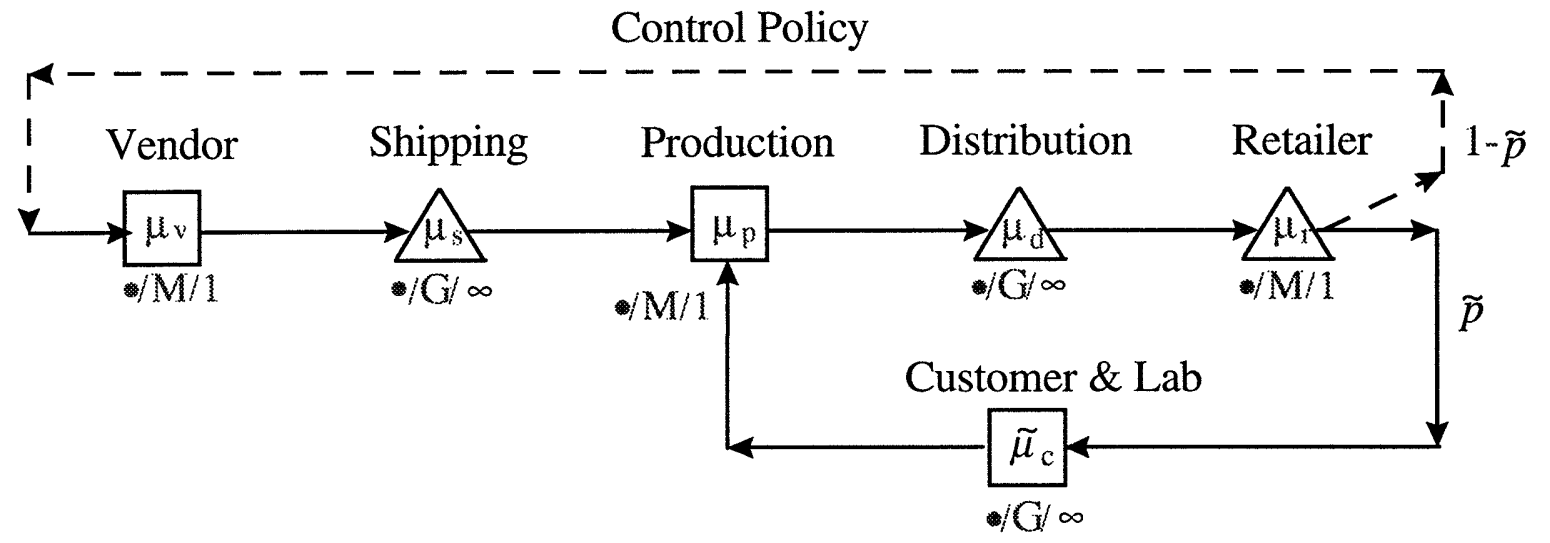

Figure 1: The queueing network model.

customer demand by a Poisson process with rate $\mu_{r}$. We assume that unsatisfied demand is lost because most single-use cameras are bought while people are traveling or on vacation. Hence, we can model this node as a single-server queue with exponential service times with rate $\mu_{r}$ : The processing time at this node corresponds to the time between demand epochs, and the idle time corresponds to the time when no cameras are available (and lost sales are incurred). To incorporate the fact that there are a number of locations where demand occurs, the retailer node could be modeled as a set of parallel queues that receives cameras which are randomly routed from the production facility. However, this would not change the nature of our results, and for simplicity we employ a single-server node.

After a camera is purchased, the customer keeps it for a certain amount of time and then takes it to a photofinishing lab to be processed. The lab is responsible for returning the camera to the production facility. We model this customer/lab node by an infinite-server queue with a general service time distribution with mean $1 / \tilde{\mu}_{c}$. We assume that a purchased camera is eventually returned to Kodak with probability $\tilde{p}$, independently of all other cameras. Hence, purchased cameras exit the system with probability $1-\tilde{p}$. (These last two parameters are unknown to the manufacturer, so we use 'tilde' to distinguish them from known parameters). Because Kodak does not incur costs at the customer/lab node, for modeling purposes it makes 
no difference whether this random routing takes place after the retailer node or after the customer/lab node. We assume that the routing occurs at the time of sale, so that the customer/lab node includes only those cameras that have been sold but will be returned. All departures from this node go to the production node.

\subsection{The Control Policy}

Even if all service time distributions in $\S 2.1$ were exponential, the derivation of an optimal procurement policy for new circuit boards would require the solution to a multi-dimensional partially-observable Markov decision process. The solution to this problem would be extremely difficult to compute and rather complex for practical implementation, so we confine our attention to a simple class of suboptimal policies. To motivate our proposed policy, we suppose for now that a centralized controller knows at the moment of a camera's purchase whether or not this camera will eventually return to Kodak. Hence, the omniscient controller can observe the six-dimensional queue length process in Figure 1. Now consider a one-for-one replenishment policy, where a new circuit board is ordered from the supplier whenever a customer buys a camera that is not eventually returned to the production facility. Under such a policy, the total number of circuit boards populating the supply chain (i.e., the aggregate base stock level) is constant and the system described in $\S 3.1$ and Figure 1 becomes a closed queueing network.

Our problem deviates from this hypothetical scenario in two ways: The number of customers at the customer/lab node is unobservable (i.e., we do not know whether a camera will be returned until it is returned) and the queueing network parameters

$\tilde{p}$ and $\tilde{\mu}_{c}$ are unknown. We develop a heuristic three-step procedure for constructing a dynamic circuit board procurement policy that adapts the one-for-one replenishment scheme to this uncertain setting. We assume that information gathering and procurement decisions are carried out periodically (e.g., weekly). Our procedure requires the following notation, where the time index and the dependence on past sales and returns are suppressed. Let $N_{i}, i=1, \ldots, 6$ denote the number of circuit boards at the vendor, shipping, production, distribution, retailer and customer/lab nodes, 
respectively, and let $N=\sum_{i=1}^{6} N_{i}$ be the total number of boards in the system. Note that the number of circuit boards at nodes 4,5 and 6 is equal to the number of cameras at these nodes, and $N_{1}, \ldots, N_{5}$ are observable but $N_{6}$ is not. Let $\tilde{\theta}$ be a vector of return flow parameters that are to be estimated from historical sales and returns data. As will be seen in $\S 3.1$, the vector $\tilde{\theta}$ essentially includes the queueing network parameters $\tilde{p}$ and $\tilde{\mu}_{c}$, but may include other parameters as well (e.g., the unobservable inventory at the beginning of the data collection period). The vector $\tilde{\theta}$ is random under a Bayesian approach, while it denotes the true state of nature in a frequentist approach; the difference will be apparent from context. Let $C(n ; \theta)$ be the expected steady-state total cost incurred in the closed queueing network in $\S 2.1$ with $n$ circuit boards, for a given value of $\tilde{\theta}$, which we denote by $\theta$. The cost function is specified in $\S 3.3$.

Our procedure consists of three steps (described in detail in $§ 3.1, \S 3.2$ and $\S 3.3$ ) that are carried out periodically. In the first step, we estimate the distribution $f_{\tilde{\theta}}(\theta)$ of the return flow parameters using information about past sales and returns. In the second step, we find $f_{N_{6} \mid \tilde{\theta}}(n \mid \theta)$, which is the distribution of the number in the unobservable portion of the system (the customer/lab node) for a given $\theta$. Note that the distribution for the total number of circuit boards in the system given $\theta$ is $f_{N \mid \tilde{\theta}}(n \mid \theta)=f_{N_{6} \mid \tilde{\theta}}\left(n-\sum_{i=1}^{5} N_{i} \mid \theta\right)$.

In the last step, we integrate the expected steady-state total cost over the distributions of the unobservable inventory and the return flow parameters, and then procure the amount that minimizes the cost. Hence, the recommended procurement quantity for new circuit boards is

$$
Q=\underset{x}{\arg \min } \int_{\theta} \int_{n} C(n+x ; \theta) f_{N \mid \tilde{\theta}}(n \mid \theta) f_{\tilde{\theta}}(\theta) d n d \theta
$$

This procedure does not result in an optimal aggregate base stock policy because the queueing network does not immediately enter a new steady state after each procurement decision; in effect we dynamically calculate the procurement quantity for a transient system using the expected total cost incurred in its steady-state counterpart. 


\section{Analysis}

\subsection{Parameter estimation}

We consider two informational structures regarding the monitoring of cameras: The trackable case, where cameras are time-stamped, and the untrackable case, where cameras are not time-stamped.

Untrackable case. The single-use camera supply chain is an example of an untrackable system. Cameras are not time-stamped, so that when a camera is returned, nothing can be inferred about its return delay. The only information available is the number of sales and returns in each time period.

The incremental nature of information received in this problem makes Bayesian estimation a natural choice. We assume that there is a discrete delay (lag) distribution, $r_{D}(d)$, governing the amount of time cameras spend with customers and at a photofinishing lab. If the probability that a camera will ever come back is $p$, and a camera was sold in period $t$, the probability it comes back in period $t+k$ is $r_{D}(k) p$. Let $n_{t}$ and $m_{t}$ denote the number of cameras sold and returned in period $t=1,2, \ldots$, respectively, where $m_{1}=0$. Then the return process can be modeled by

$$
m_{t}=r_{D}(1) p n_{t-1}+r_{D}(2) p n_{t-2}+\ldots r_{D}(t-1) p n_{1}+\epsilon_{t} \quad t=2, \ldots
$$

where $\epsilon_{t} \sim N\left(0, \sigma^{2}\right)$. This type of relation is referred to as a distributed lag model in the literature (e.g., Zellner 1987). Usually, a specific form of distribution involving one or two parameters is assumed for the lag, which reduces the number of parameters to be estimated.

In the Appendix, we illustrate the Bayesian estimation procedure with a geometrically distributed lag with parameter $\tilde{q}$ (the probability a sold camera is returned in the next period, given it will be returned). We then show how this extends to a Pascal distribution, which allows more flexibility in the shape of the delay distribution. We also show how to carry out hypothesis testing of different lag models.

The data available from Kodak consists of 22 months of sales and returns. We used the above method on data with a geometric lag to find a posterior density for 
return flow parameters $\tilde{\theta}=(\tilde{p}, \tilde{q})$. The point estimates $\hat{p}$ and $\hat{q}$ (derived from the joint density function in the Appendix) obtained after 22 months were found to be 0.5 and 0.58 , respectively. (The data has been disguised so that $\hat{p}=0.5$.) The hypotheses of geometric, Pascal lag one and Pascal lag two were tested, taking prior odds ratios of 1 (i.e., all hypothesis are assumed to be equally likely). The posterior probabilities thus obtained were $\pi_{1}=0.977, \pi_{2}=0.022$ and $\pi_{3}=0.001$, and hence a geometric lag model seems justified. This conclusion is not surprising because many single-use cameras are typically bought for some occasion, and are used and returned quickly after the sale, which is consistent with a geometric distribution. Hereafter, we assume that the delay is geometric; in this case, the unknown queueing network parameter $\tilde{\mu}_{c}$ corresponds to the geometric parameter $\tilde{q}$.

One issue that arises with this set of data is that of initial conditions. The above analysis assumes that all returned cameras were sold during the data collection period, whereas the Kodak system had been in operation for some time when the data collection began. Therefore, some of the returns in the initial months depend on sales that occurred prior to data collection. To assess the impact of initial conditions, we employ the maximum likelihood estimation method, which allows us to estimate the number of unobserved cameras at the beginning of the period, and therefore avoids the initialization problem. Let $\tilde{n}_{0}$ denote the number of cameras that have not been returned at the time data collection begins. Using the same notation as above, for a given return probability value $p$, a given geometric delay parameter value $q$, and an outstanding number of cameras $n_{0}$, we model the returns as

$$
m_{t}=p q n_{t-1}+p q(1-q) n_{t-2}+\ldots+p q(1-q)^{t-1} n_{0}+u_{t}, t=2, \ldots,
$$

where $m_{1}=p q n_{0}+u_{1}$ and $u_{t} \sim N\left(0, \sigma^{2}\right)$. Using a maximum likelihood estimation method developed in Dhrymes (1985), we find estimates for $\tilde{\theta}=\left(\tilde{p}, \tilde{q}, \tilde{n}_{0}, \tilde{\sigma}^{2}\right)$ for the Kodak data. The resulting point estimates are $\hat{p}=0.5$ and $\hat{q}=0.55$, which are very close to the estimates obtained from Bayesian estimation. As expected, $\hat{q}$ is lower with this method, but the small difference suggests that the effect of initial conditions is indeed minimal. 
Trackable Case. This situation arises when the manufacturer time-stamps the product at the time of purchase, $t_{\text {sale }}$. At any given time $t$, some of the cameras will have been returned. For these cameras, we can record how much time was spent outside the system. For others, we know that their delay is longer than $t-t_{\text {sale }}$. In the survival analysis literature, this kind of data is referred to as right-censored survival data. In the Appendix we use the expectation maximization (EM) algorithm (Dempster, Laird and Rubin 1977) to estimate the return flow parameters $\tilde{\theta}=(\tilde{p}, \tilde{q})$ in the geometric delay case.

There are several problems associated with the trackable case. Products are more apt to be time-stamped with the date of production, not the date of sale. In either case, the data collection requirements are greater than in the untrackable case. Indeed, this kind of data was not available from Kodak. In $\S 4$, we assess whether this additional information is worth collecting. Finally, the EM algorithm only produces a point estimate $\hat{\theta}$, not a distribution; hence, we let $f_{\tilde{\theta}}(\theta)$ have a unit impulse at $\hat{\theta}$ in our three-step procedure.

\subsection{Estimating the Unobservable Inventory}

Untrackable Case. At time $t$, we know the number of sales $n_{i}$ and the number of returns $m_{i}$ in periods $i=1,2, \ldots, t$. We also have an estimate of the return probability and return delay distribution. Let $W_{i}$ be the number of circuit boards that will be returned in periods $[t+1, t+H]$ from the sales in period $i, i \leq t$, for some time horizon $H$. Kelle and Silver (1989a) show that $\left(\sum_{i=1}^{t} W_{i}, m_{t}, m_{t-1}, \ldots, m_{2}\right)$ can be approximated by a multivariate normal vector. Its mean and covariance matrix depend on covariances between returns in different periods. Under this approximation, the conditional distribution of $\left(\sum_{i=1}^{t} W_{i} \mid m_{t}, m_{t-1}, \ldots, m_{2}\right)$ is also normal and its mean and variance are easily calculated.

We wish to calculate the distribution of $N_{6}(t)$, the number in the customer/lab node in period $t$. If we let the time horizon $H=\infty$, then $N_{6}(t)=\sum_{i=1}^{t} W_{i}$ and applying Kelle and Silver's method gives the desired distribution.

Trackable case. This method is also adapted (to the infinite horizon setting) 
from Kelle and Silver (1989). Let $p_{i}$ denote the probability that a sale in period $i$, which has not been returned by period $t$, will eventually be returned. For a given return probability value $p$ and a given geometric delay parameter value $q$, $p_{i}=\frac{p(1-q)^{t-i+1}}{1-p+p(1-q)^{t-i+1}}$. Let $n_{i}$ denote the number of cameras sold in period $i$, and $r_{i}$ denote the number of these cameras that have already been returned (by period $t$ ). In period $t$, the conditional expectation and variance of the amount of unobservable inventory due to sales in period $i$ are $\left(n_{i}-r_{i}\right) p_{i}$ and $\left(n_{i}-r_{i}\right) p_{i}\left(1-p_{i}\right)$, respectively. Because the $n_{i}-r_{i}$ 's are mutually independent, the expectation and variance of the total unobservable inventory at time $t$ are computed by summing the above quantities over $i=1, \ldots, t$. A normal distribution with this mean and variance is then used to approximate the distribution of the unobservable inventory.

\subsection{Calculating the Expected Steady-state Cost}

The third step of our procedure requires the calculation of $C(n ; \theta)$, which is the expected steady-state total cost incurred by the closed queueing network in $\S 2.1$ with $n$ circuit boards, for a given value of $\tilde{\theta}=\theta$. Recalling that $\tilde{\theta}$ specifies $\tilde{p}$ and $\tilde{q}$, and that $\tilde{q}=\tilde{\mu}_{c}$ under our geometric delay assumption, we let $\theta$ correspond to $\left(p, \mu_{c}\right)$. Although the closed queueing network has a product-form stationary distribution, it requires the calculation of a normalization constant, which is somewhat tedious in the context of our three-step procedure. In order to derive a closed-form expression for $C(n ; \theta)$, we resort to the fixed population mean (FPM) approximation developed by Whitt (1984). To use this method, we transform the closed network in Figure 1 into an open network by letting departures from a decoupling infinite-server node (the customer/lab node, in our case) exit the system and creating external Poisson arrivals (at an unspecified rate $\lambda_{c}$ ) to the production node. The FPM method approximates the throughput rate in the closed system with $n$ circuit boards by the external arrival rate to the open network which makes the expected number in that system equal to $n$. The accuracy of the FPM approximation in our setting is investigated in $\S 4.2$.

Let $n_{i}^{o}\left(\lambda_{c}\right)$ be the expected steady-state number of circuit boards in this open 
network at node $i=1, \ldots, 6$ when the external arrival rate to the production node is $\lambda_{c}$, and let $n^{o}\left(\lambda_{c}\right)=\sum_{i=1}^{6} n_{i}^{o}\left(\lambda_{c}\right)$. Since our queueing network is of product form, we have

$$
n^{o}\left(\lambda_{c}\right)=\lambda_{c}\left(\frac{1-p}{p \mu_{v}-(1-p) \lambda_{c}}+\frac{1-p}{p \mu_{s}}+\frac{1}{p \mu_{p}-\lambda_{c}}+\frac{1}{p \mu_{d}}+\frac{1}{p \mu_{r}-\lambda_{c}}+\frac{1}{\mu_{c}}\right),
$$

which is a fourth-order equation in $\lambda_{c}$. The FPM approximation requires us to find the value of $\lambda_{c}$ such that $n^{o}\left(\lambda_{c}\right)=n$. To obtain a closed-form solution to this equation, we omit several of the terms in (2). In particular, the vendor node and the production node in the Kodak system have very small queue lengths compared to the other four nodes, so we approximate (2) by

$$
n^{o}\left(\lambda_{c}\right) \simeq \frac{\lambda_{c}(1-p)}{p \mu_{s}}+\frac{\lambda_{c}}{p \mu_{d}}+\frac{\lambda_{c}}{p \mu_{r}-\lambda_{c}}+\frac{\lambda_{c}}{\mu_{c}}
$$

which is quadratic in $\lambda_{c}$. Using (3) to solve $n^{o}\left(\lambda_{c}\right)=n$ gives

$$
\lambda_{c}(n)=\frac{p\left(C_{1} n+\mu_{r} C_{2}+C_{1}-\sqrt{\left(C_{1} n-\mu_{r} C_{2}+C_{1}\right)^{2}+4 \mu_{r} C_{1} C_{2}}\right)}{2 C_{2}},
$$

where $C_{1}=\mu_{d} \mu_{c} \mu_{s}$ and $C_{2}=p \mu_{d}\left(\mu_{s}-\mu_{c}\right)+\mu_{c}\left(\mu_{s}+\mu_{d}\right)$.

Now we calculate the expected steady-state total cost per unit time, which incorporates lost sales ( $c_{l}$ per camera), procurement ( $c_{v}$ per board) and holding inventory at the retailer and distribution nodes ( $c_{h}$ per board per unit time). For an open queueing network with arrival rate $\lambda_{c}$, this cost is

$$
C^{o}\left(\lambda_{c}\right)=\frac{(1-p) \lambda_{c} c_{v}}{p}+\left(1-\frac{\lambda_{c}}{p \mu_{r}}\right) \mu_{r} c_{l}+c_{h}\left(\frac{\lambda_{c}}{p \mu_{d}}+\frac{\lambda_{c}}{p \mu_{r}-\lambda_{c}}\right) .
$$

By the FPM method, the expected steady-state $\operatorname{cost} C(n ; \theta)$ associated with a given population level $n$ is approximated in closed form by $C^{0}\left(\lambda_{c}(n)\right)$ using (4) and (5).

\section{Computational Study}

This section contains a simulation experiment aimed at evaluating the effectiveness of our policy, and determining the key controllable and uncontrollable factors that affect system performance. We describe the design of the study in $\S 4.1$ and present and discuss the results in $\S 4.2$. 


\subsection{Experimental Design}

In this subsection we describe the model parameters, the policies and the initialization scheme.

Parameter Values. The parameter values in our simulation model are roughly representative of the Kodak environment. We set the true return probability $\tilde{p}=0.5$, procurement cost $c_{v}=\$ 5 /$ unit, lost sales cost $c_{l}=\$ 10 /$ unit and inventory holding cost $c_{h}=\$ 0.02 /$ unit/week. The delays at the shipping and distribution nodes are exponential with means of $\mu_{s}^{-1}=8$ and $\mu_{d}^{-1}=2$, respectively. The customer/lab delay is also exponential with true mean $\tilde{\mu}_{c}^{-1}=8$ weeks. The service rates at the circuit board vendor, production and retailer nodes are $\mu_{v}=20,000, \mu_{p}=20,000$ and $\mu_{r}=18,000$ per week, respectively.

Policies. To put the performance of our proposed policy into perspective, we simulate five different policies. These policies, as well as the informational structures under which they operate, are summarized in Table 1. Recall that at the beginning of each period, the following sequence is carried out using the data to date: Estimate the return flow parameters, estimate the distribution of unobservable inventory and determine the recommended procurement quantity.

\begin{tabular}{|c||c|c|c|c|}
\hline \multirow{2}{*}{\multicolumn{1}{|c||}{ Policy }} & Informational Structure/Parameter Estimation & Recommended \\
\cline { 2 - 4 } & $\begin{array}{c}\text { Return Flow } \\
\text { Parameters }\end{array}$ & $\begin{array}{c}\text { Customer/Lab } \\
\text { Inventory }\end{array}$ & $\begin{array}{c}\text { Data } \\
\text { Collection }\end{array}$ & $\begin{array}{c}\text { Procurement } \\
\text { Quantity }\end{array}$ \\
\hline \hline CLAIRVOYANT & Known & Observable & - & Equation (7) \\
\hline TRACKABLE & EM Algorithm & Kelle-Silver & By Camera & Equation $(1)$ \\
\hline UNTRACKABLE & Distributed Lag & Kelle-Silver & Periodic & Equation $(1)$ \\
\hline UNTRACKABLE $\left(\hat{p}, \hat{\mu}_{c}\right)$ & Guess $\left(\hat{p}, \hat{\mu}_{c}\right)$ & Kelle-Silver & Periodic & Equation $(1)$ \\
\hline OBSERVABLE & Distributed Lag & Ignored & Periodic & Equation $(9)$ \\
\hline
\end{tabular}

Table 1: Description of the simulated policies.

The first policy, referred to as CLAIRVOYANT in Table 1, assumes that the true values of the parameters $\tilde{p}$ and $\tilde{\mu}_{c}$ and the current number of customers in the 
customer/lab node are known, and uses the optimal aggregate base stock level from the FPM approximation. This base stock level is given by $n^{o}\left(\lambda_{c}^{*}\right)$ in (3) (with $\tilde{p}$ and $\tilde{\mu}_{c}$ in place of $p$ and $\mu_{c}$ ), where

$$
\lambda_{c}^{*}=\tilde{p}\left(\mu_{r}-\sqrt{\frac{c_{h} \mu_{r}}{c_{l}-(1-\tilde{p}) c_{v}-c_{h} / \mu_{d}}}\right)
$$

is the arrival rate that minimizes the cost (with $\tilde{p}$ in place of $p$ ) in (5). Hence, the recommended procurement quantity is

$$
Q=n^{o}\left(\lambda_{c}^{*}\right)-\sum_{i=1}^{6} N_{i}
$$

The next two policies, TRACKABLE and UNTRACKABLE, are our proposed policies in equation (1) corresponding to these two informational structures.

UNTRACKABLE $\left(\hat{p}, \hat{\mu}_{c}\right)$ is identical to the UNTRACKABLE policy, except that the joint distribution of the return probability $\tilde{p}$ and the return delay parameter $\tilde{\mu}_{c}$ derived in $\S 3.1$ is replaced by a unit impulse at the point estimate $\hat{\theta}=\left(\hat{p}, \hat{\mu}_{c}\right)$. In $\S 4.2$, we assess the importance of estimating $\tilde{p}$ and $\tilde{\mu}_{c}$ by varying the value of these two estimates.

The last policy in Table 1, OBSERVABLE, allows us to isolate the importance of estimating the unobservable inventory, $N_{6}$. This policy does not attempt to estimate $N_{6}$ or the return delay parameter $\tilde{q}$, and uses a base stock policy based only on the observable portion of the supply chain, $\sum_{i=1}^{5} N_{i}$. To derive this policy, we consider an open queueing network that is identical to the first five nodes in Figure 1, except all departures from node 5 (the retailer node) exit the system, and there are exogenous Poisson arrivals to node 1 (the vendor) and node 3 (the production facility) with rates $\lambda_{1}$ and $p \mu_{r}$, respectively. The distribution $f_{\tilde{p}}(p)$ of the random variable $\tilde{p}$ is estimated using the distributed lag model in $\S 3.1$. Using the same cost structure as in $\S 3.2$, we find the cost-minimizing value of $\lambda_{1}$ for a given return probability $p$ to be

$$
\lambda_{1}^{*}(p)=(1-p) \mu_{r}-\sqrt{\frac{c_{h} \mu_{r}}{c_{l}-c_{v}-c_{h} / \mu_{d}}} .
$$

For a given return probability $p$, we use the FPM logic in reverse, and set the target base stock level equal to the expected number of customers in the open queueing 
network with arrival rate $\lambda_{1}^{*}(p)$ :

$$
\frac{\lambda_{1}^{*}(p)}{\mu_{v}-\lambda_{1}^{*}(p)}+\frac{\lambda_{1}^{*}(p)}{\mu_{s}}+\frac{\lambda_{1}^{*}(p)+p \mu_{r}}{\mu_{p}-\lambda_{1}^{*}(p)-p \mu_{r}}+\frac{\lambda_{1}^{*}(p)+p \mu_{r}}{\mu_{d}}+\frac{\lambda_{1}^{*}(p)+p \mu_{r}}{(1-p) \mu_{r}-\lambda_{1}^{*}(p)} .
$$

We denote the quantity in (8) by $N\left(\lambda_{1}^{*}(p)\right)$. Integrating this base stock level over the density $f_{\tilde{p}}(p)$ yields the recommended procurement quantity for the OBSERVABLE policy:

$$
Q=\int_{p} N\left(\lambda_{1}^{*}(p)\right) f_{\tilde{p}}(p) d p-\sum_{i=1}^{5} N_{i} .
$$

Implementation. For each of the five policies in Table 1, the system is started empty and the review period is one week. Initially, the vendor node works at full capacity and pushes items through the system. Finished goods inventory is collected at the retailer node until it reaches the level $\mu_{r}$, the expected demand for one week. At this point, sales are allowed to start. This design allows a meaningful comparison of systems with different shipping delays and sales rates.

Three periods after returns start, the parameter and unobservable inventory estimation procedures are carried out if necessary, and a control policy is used to determine procurement decisions. The three-period restriction is necessary for cases where estimation is required, and was enforced in all policies in Table 1 to ensure consistency. For all policies, the actual procurement quantity $Q^{*}$ equals the smallest integer greater than or equal to the recommended procurement quantity $Q$ in Table 1 , $\lceil Q\rceil$, if $0 \leq Q \leq 1.5 \mu_{v}$; otherwise, $Q^{*}=0$ if $Q<0$ and $Q^{*}=1.5 \mu_{v}$ if $Q>1.5 \mu_{v}$. This upper bound is imposed so that overestimation of the procurement quantity in a given period does not adversely affect performance over an extended period. The factor 1.5 ensures that for all parameter values used in our simulations, this bound is larger than four standard deviations above the mean quantity processed at the vendor in one period.

Cost collection starts after the three-period restriction, and includes procurement costs, lost sales costs, and inventory holding costs at the production, distribution and retailer nodes. The system is simulated for 78 weeks (the product life cycle is approximately 1.5 years) and 30 simulation runs are carried out for each scenario. For 
each run, the same initial random number seed is used across all policies to reduce the variance in pairwise comparisons of policies.

\subsection{Results}

We first report the main simulation results, and then perform a sensitivity analysis with respect to several key parameters.

Main Results. The costs for the simulated policies are presented in Table 2. Observations 2 through 5 below can be extracted from this table.

\begin{tabular}{|c|c|}
\hline Policy & Cost $(\$ 1000 /$ week $)$ \\
\hline \hline CLAIRVOYANT & $48.90( \pm 0.04)$ \\
\hline TRACKABLE & $48.90( \pm 0.04)$ \\
\hline UNTRACKABLE & $48.94( \pm 0.03)$ \\
\hline UNTRACKABLE $(0.5,0.125)$ & $48.93( \pm 0.04)$ \\
\hline UNTRACKABLE $(0.4,0.125)$ & $50.55( \pm 0.09)$ \\
\hline UNTRACKABLE $(0.6,0.125)$ & $59.30( \pm 0.17)$ \\
\hline UNTRACKABLE $(0.5,0.083)$ & $49.07( \pm 0.09)$ \\
\hline UNTRACKABLE $(0.5,0.25)$ & $53.11( \pm 0.17)$ \\
\hline OBSERVABLE & $59.50( \pm 0.14)$ \\
\hline
\end{tabular}

Table 2: The simulated average cost (and $95 \%$ confidence intervals) for nine policies.

1. The accuracy of the FPM approximation. A simulation study (not appearing in Table 2) was undertaken to assess the accuracy of the FPM approximation. For the parameter values given in $\S 4.1$ (except that the mean shipping delay was two weeks rather than eight weeks), the FPM-estimated cost at the FPM base stock level (given by $n^{o}\left(\lambda_{c}^{*}\right)$ in (7)) was $0.12 \%$ higher than the simulated cost at the FPM base stock level. Hence, the FPM approximation is very accurate in our setting.

2. The impact of estimating $\tilde{p}$ and $\tilde{\mu}_{c}$. Comparing the five $\operatorname{UNTRACKABLE}\left(\hat{p}, \hat{\mu}_{c}\right)$ policies in Table 2, we see that a $20 \%$ error in estimating the true return probability leads to a cost increase of $3.3 \%(\hat{p}=0.4)$ and $21.2 \%(\hat{p}=0.6)$. Underestimating the 
true mean delay by a factor of two causes a $8.5 \%$ cost increase and overestimating it by $50 \%$ results in only a $0.3 \%$ cost increase. Overestimating $\tilde{p}$ or $\tilde{\mu}_{c}$ results in lost sales, while underestimating these quantities generates superfluous inventory. Thus, overestimation, particularly of the return probability $\tilde{p}$, is more costly. In summary, the most costly error is to overestimate $\tilde{p}$.

3. The accuracy of estimating $N_{6}$. The only difference between the UNTRACKABLE $(0.5,0.125)$ policy and the CLAIRVOYANT policy is that the former estimates $N_{6}$ and the latter observes $N_{6}$. The minute difference in performance between these two policies shows that the Kelle-Silver method provides an accurate estimate for $N_{6}$ in the untrackable case.

4. The impact of estimating $N_{6}$. There are two differences between the UNTRACKABLE policy and the OBSERVABLE policy: The latter policy does not estimate the return delay $\tilde{q}$ or the unobservable inventory $N_{6}$. Observation 2 above shows that the UNTRACKABLE policy is relatively insensitive to the estimate $\hat{q}$. Hence, the $21.6 \%$ cost difference between these two policies suggests that it is important to estimate $N_{6}$.

5. The accuracy and impact of tracking cameras. The proximity in cost of the TRACKABLE and UNTRACKABLE policies suggests that the economic gains from tracking cameras is small, and is probably outweighed by the cost of tracking. Both of these policies perform nearly as well as the UNTRACKABLE $(0.5,0.125)$ policy, which shows that the EM algorithm and the distributed lag method work well.

Sensitivity Analysis. A strategic issue facing Kodak is whether to reduce the long shipping delay, by either using air shipments or switching to a domestic supplier. We repeated all the simulations in Table 2, but with a shipping delay of two weeks rather than eight weeks. Not surprisingly, the controllability of the system improves when the shipping delay is shorter, and the costs (we do not consider the cost required to reduce this shipping delay) decrease. The average cost reduction for the nine policies in Table 2 is $5.3 \%$ per week, which is less than the cost reductions gained by estimating the return probability $\tilde{p}$ or the unobservable inventory $N_{6}$ in an intelligent manner. However, the cost reductions for UNTRACKABLE(0.6,0.125) 
and UNTRACKABLE $(0.5,0.25)$ are $10.9 \%$ and $9.9 \%$, respectively. Therefore, in cases where parameters are overestimated, a short shipping delay can significantly improve system performance.

Our simulation model used a shipping delay, demand rate and time horizon (18 months) to reflect the scenario of Kodak's single-use camera. To assess the robustness of our three main observations from Table 2 (it is important to estimate $N_{6}$ and not overestimate $\tilde{p}$, but it is not worthwhile tracking cameras), we compute in Table 3 the cost ratios of several policies for two values of the shipping delay (two and eight weeks), three values of the vendor rate $\mu_{v}$, the production rate $\mu_{p}$, and the demand rate $\mu_{r}$ (the base rates, 0.1 of these rates and 0.01 of these rates), and four values of the product life-cycle length (i.e., the number of weeks that costs were collected).

The difference in performance between UNTRACKABLE and TRACKABLE observed in Table 3 is primarily due to the difference in the rate of convergence of the parameter estimates found using the distributed lags model and the EM algorithm, respectively. In general, the EM algorithm converges more rapidly to the true unknown parameter values and thus achieves better performance for small sample sizes (low total demand volume over product life cycle). Therefore, tracking inventory provides significant relative benefits only if the system is in transience throughout the product life cycle in the sense that the parameter estimates have not yet converged. As the total demand volume over the product life cycle increases, the same system performance can be achieved with less information (without tracking the cameras).

The UNTRACKABLE(0.6,0.125)/UNTRACKABLE(0.5,0.125) results illustrate the impact of delays on system performance. Since lost sales lag behind procurement decisions by several weeks, overestimating the return probability and ordering less than necessary can lead to better initial performance. However, as the life cycle increases, the importance of correct estimation becomes apparent, particularly in the long shipping delay scenarios.

The results for OBSERVABLE/UNTRACKABLE show that the impact of estimating the unobservable inventory is significant. In the low volume, short shipping delay case, OBSERVABLE dominates UNTRACKABLE due to the lag between pro- 
curement and lost sales, as above. For all other cases, however, UNTRACKABLE dominates OBSERVABLE, especially in the long shipping delay case.

A caveat of the above analysis is that it does not include end-of-life-cycle effects (e.g., decreasing demand rate, cost of ending inventory). However, the qualitative nature of our conclusions should not be affected by this.

\begin{tabular}{|c|c|c|c|c|c|c|}
\hline \multirow{2}{*}{$\begin{array}{c}\text { Shipping } \\
\text { Delay }\end{array}$} & \multirow{2}{*}{$\begin{array}{c}\text { Demand } \\
\text { Rate }\end{array}$} & \multirow{2}{*}{$\begin{array}{c}\text { Policies } \\
\text { Compared }\end{array}$} & \multicolumn{4}{|c|}{ Product Life Cycle (weeks) } \\
\hline & & & 16 & 32 & 48 & 64 \\
\hline \multirow{9}{*}{ Short } & \multirow{3}{*}{ Low } & $\frac{\text { UNTRACKABLE }}{\text { TRACKABLE }}$ & $151.3 \%$ & $102.3 \%$ & $101.0 \%$ & $101.0 \%$ \\
\hline & & $\begin{array}{l}\text { UNTRACKABLE }(0.6,0.125) \\
\text { UNTRACKABLE }(0.5,0.125) \\
\text { UNTREST }\end{array}$ & $94.8 \%$ & $96.1 \%$ & $97.1 \%$ & $97.7 \%$ \\
\hline & & $\begin{array}{l}\text { OBSERVABLE } \\
\text { UNTRACKABLE } \\
\end{array}$ & $58.8 \%$ & $93.2 \%$ & $95.6 \%$ & $96.5 \%$ \\
\hline & \multirow{3}{*}{ Medium } & $\frac{\text { UNTRACKABLE }}{\text { TRACKABLE }}$ & $107.5 \%$ & $100.8 \%$ & $100.6 \%$ & $100.4 \%$ \\
\hline & & $\begin{array}{l}\text { UNTRACKABLE }(0.6,0.125) \\
\operatorname{UNTRACKABLE}(0.5,0.125) \\
\operatorname{UNTRE}\end{array}$ & $94.4 \%$ & $98.2 \%$ & $101.5 \%$ & $103.2 \%$ \\
\hline & & $\frac{\text { OBSERVABLE }}{\text { UNTRACKABLE }}$ & $93.8 \%$ & $104.9 \%$ & $104.9 \%$ & $104.5 \%$ \\
\hline & \multirow{3}{*}{ High } & $\frac{\text { UNTRACKABLE }}{\text { TRACKABLE }}$ & $102.1 \%$ & $100.7 \%$ & $100.4 \%$ & $100.3 \%$ \\
\hline & & $\frac{\text { UNTRACKABLE }(0.6,0.125)}{\operatorname{UNTRACKABLE}(0.5,0.125)}$ & $94.1 \%$ & $104.6 \%$ & $109.2 \%$ & $111.5 \%$ \\
\hline & & $\frac{\text { OBSERVABLE }}{\text { UNTRACKABLE }}$ & $108.9 \%$ & $118.6 \%$ & $120.0 \%$ & $120.3 \%$ \\
\hline \multirow{9}{*}{ Long } & \multirow{3}{*}{ Low } & $\frac{\text { UNTRACKABLE }}{\text { TRACKABLE }}$ & $133.1 \%$ & $100.8 \%$ & $100.3 \%$ & $100.4 \%$ \\
\hline & & $\begin{array}{l}\text { UNTRACKABLE }(0.6,0.125) \\
\text { UNTRACKABLE }(0.5,0.125)\end{array}$ & $90.4 \%$ & $94.1 \%$ & $96.6 \%$ & $97.7 \%$ \\
\hline & & $\frac{\text { OBSERVABLE }}{\text { UNTRACKABLE }}$ & $74.6 \%$ & $105.0 \%$ & $104.5 \%$ & $103.3 \%$ \\
\hline & \multirow{3}{*}{ Medium } & $\frac{\text { UNTRACKABLE }}{\text { TRACKABLE }}$ & $109.7 \%$ & $5 \%$ & $100.4 \%$ & $100.3 \%$ \\
\hline & & $\begin{array}{l}\text { UNTRACKABLE }(0.6,0.125) \\
\text { UNTRACKABLE }(0.5,0.125) \\
\end{array}$ & $97.7 \%$ & $106.8 \%$ & $111.0 \%$ & $113.5 \%$ \\
\hline & & $\frac{\text { OBSERVABLE }}{\text { UNTRACKABLE }}$ & $105.9 \%$ & $119.5 \%$ & $116.2 \%$ & $113.7 \%$ \\
\hline & \multirow{3}{*}{ High } & $\frac{\text { UNTRACKABLE }}{\text { TRACKKABLE }}$ & $100.8 \%$ & $100.2 \%$ & $100.1 \%$ & $100.1 \%$ \\
\hline & & $\begin{array}{l}\text { UNTRACKABLE }(0.6,0.125) \\
\operatorname{UNTRACKABLE}(0.5,0.125) \\
\end{array}$ & $102.9 \%$ & $113.2 \%$ & $118.1 \%$ & $120.8 \%$ \\
\hline & & $\frac{\text { OBSERVABLE }}{\text { UNTRACKABLE }}$ & $121.8 \%$ & $127.1 \%$ & $124.7 \%$ & $122.2 \%$ \\
\hline
\end{tabular}

Table 3: Relative costs of policies under various scenarios. 


\section{Conclusion}

Environmental concerns, legislative actions and increasing product disposal costs have led many firms to adopt "green manufacturing" practices, such as the recovery and remanufacturing of used products. These practices lead to challenging reverse logistics problems, where the return flows of used products need to be taken into account. We develop and analyze a model of the supply chain for Kodak's single-use camera, from the overseas production of circuit boards to the photofinishing lab's development of the film and subsequent return of the camera to Kodak's production facility. The model and analysis have two distinctive features: We use a queueing network model, which allows for considerable flexibility in modeling production and distribution facilities within the supply chain, and we focus on the statistical aspects of the problem, by dynamically estimating the probability that sold cameras are returned, the delay of returned cameras, and the number of cameras that have been sold and will be returned at some future time. Although the paper is written in a problem-specific manner, the model and methods used here are generic and can be adapted to other remanufacturing settings.

Our framework allows us to understand the dominant characteristics of the system and provide guidelines for managing it. The two most important operational levers appear to be the accurate dynamic estimation (using historical sales and returns data) of the number of cameras that have been sold and will be returned at a future date and of the return probability of a camera. The former is important at all life cycles we consider, while the latter becomes more important as the life cycle increases. An important strategic lever is the use of a domestic versus overseas vendor, which results in short or long shipping delays, respectively. A long shipping delay leads to a less controllable system, and causes a relative deterioration in performance of policies not making good use of the available information. Our analysis also allows us to distinguish when it is important to use information-intensive monitoring of a product. If the total demand volume for a product over its life cycle is low, significant relative benefits can be gained from using the additional information obtained by time- 
stamping the product. As this quantity increases, the relative benefit of collecting additional information diminishes and a less information-intensive method becomes adequate.

Although our model possesses some features rarely found in inventory models, it also lacks two characteristics that are common in inventory models: We ignore a fixed ordering cost for new circuit boards, and assume a rather simple (one-parameter) form of policy. Approximations for queueing networks with batch arrivals (e.g., Whitt 1994) might allow for the derivation of an aggregate $(s, S)$ policy, but the analysis of a policy that depends on a multi-dimensional state variable would likely be more difficult.

Three other issues deserve further investigation. First, our model assumes that unsatisfied demand is lost, and our analysis does not obviously extend to the backorder case. Also, Kodak's demand has seasonal (sales for flash cameras are highest in the winter holiday season) and life-cycle components that were ignored in our analysis. Finally, customer demand represents the largest source of parameter uncertainty in many systems, and statistical techniques for demand estimation in the lost sales case (e.g., Nahmias 1994, Lariviere and Porteus 1995) can be incorporated into our framework.

\section{Acknowledgment}

We thank Bruce Alexander, Tris Munz, Steve Rumsey and Al van de Moere for sharing information and data about Kodak's single-use camera.

\section{Appendix}

\section{Bayesian Estimation for the Distributed Lag Model}

Recall that in $\S 3.1$, we modeled the dependence of sales on returns by the relation

$$
m_{t}=p q n_{t-1}+p q(1-q) n_{t-2}+p q(1-q)^{2} n_{t-3}+\ldots+\epsilon_{t} \quad \text { for } \quad t=2,3, \ldots
$$

where $\epsilon_{t} \sim N\left(0, \sigma^{2}\right)$. Suppose that data is available for the first $T$ periods. Subtracting $(1-q) m_{t}$ from both sides of the above relation, we get

$$
m_{t}=(1-q) m_{t-1}+p q n_{t-1}+\epsilon_{t}-(1-q) \epsilon_{t-1} \quad t=2,3, \ldots, T
$$


which is the form to be used in the analysis. Let $\mathbf{u}=\left(u_{2}, u_{3}, \ldots, u_{T}\right)$ where $u_{t}=$ $\epsilon_{t}-(1-q) \epsilon_{t-1}$. The covariance matrix for the error term is $E\left(\mathbf{u u}^{\prime}\right)=\sigma^{2} G$, where $G$ is the $(T-1) \times(T-1)$ matrix

$$
G=\left(\begin{array}{ccccc}
1+(1-q)^{2} & -(1-q) & 0 & . & 0 \\
-(1-q) & 1+(1-q)^{2} & -(1-q) & . . & 0 \\
: & : & : & : & : \\
0 & 0 & 0 & -(1-q) & 1+(1-q)^{2}
\end{array}\right)
$$

The joint density function for $\mathbf{m}=\left(m_{2}, m_{3}, \ldots, m_{T}\right)$ is

$f\left(\mathbf{m} \mid p, q, \sigma, m_{1}\right) \propto \frac{|G|^{-1 / 2}}{\sigma^{T}} \exp \left[-\frac{1}{2 \sigma^{2}}\left(\mathbf{m}-(1-q) \mathbf{m}_{-1}-p q \mathbf{n}\right)^{\prime} G^{-1}\left(\mathbf{m}-(1-q) \mathbf{m}_{-1}-p q \mathbf{n}\right)\right]$.

If we take the prior density for the parameters of the model to be $f(p, q, \sigma) \propto \frac{1}{\sigma}$, which corresponds to a diffuse prior, then the posterior density becomes $f\left(p, q, \sigma \mid \mathbf{m}, m_{1}\right) \propto \frac{|G|^{-1 / 2}}{\sigma^{T+1}} \exp \left[-\frac{1}{2 \sigma^{2}}\left(\mathbf{m}-(1-q) \mathbf{m}_{-1}-p q \mathbf{n}\right)^{\prime} G^{-1}\left(\mathbf{m}-(1-q) \mathbf{m}_{-1}-p q \mathbf{n}\right)\right]$. Integrating with respect to $\sigma$, we obtain

$$
f\left(p, q \mid \mathbf{m}, m_{1}\right) \propto \frac{|G|^{-1 / 2}}{\left[\left(\mathbf{m}-(1-q) \mathbf{m}_{-1}-p q \mathbf{n}\right)^{\prime} G^{-1}\left(\mathbf{m}-(1-q) \mathbf{m}_{-1}-p q \mathbf{n}\right)\right]^{T / 2}} .
$$

The normalizing constant can be calculated to determine the joint posterior density function of $\tilde{p}$ and $\tilde{q}$.

The estimation procedure for the Pascal distribution is identical except for changes in the expression relating $m_{t}, m_{t-1}$ and $n_{t-1}$, and consequently the matrix $G$. For Pascal of order two, we obtain

$$
m_{t}=2(1-q) m_{t-1}-(1-q)^{2} m_{t-2}+p q^{2} n_{t-2}+u_{t} \quad t=3,4, \ldots, T
$$

where $u_{t}=\epsilon_{t}-2(1-q) \epsilon_{t-1}+(1-q)^{2} \epsilon_{t-2}$, and $G$ is a symmetric $(T-2) \times(T-2)$ matrix whose nonzero entries are of the form $E\left(u_{k}^{2}\right)=1+4(1-q)^{2}+(1-q)^{4}, E\left(u_{k} u_{k+1}\right)=$ $E\left(u_{k+1} u_{k}\right)=-2(1-q)\left(1+(1-q)^{2}\right)$ and $E\left(u_{k} u_{k+2}\right)=E\left(u_{k+2} u_{k}\right)=(1-q)^{2}$.

It is also possible to compare different distributed lag models by assigning prior odds ratios and determining posterior odds ratios, from which posterior probabilities 
associated with the models can be computed. For example, let us consider three alternative models for the delays: Geometric $\left(H_{1}\right)$, Pascal of lag two $\left(H_{2}\right)$ and Pascal of lag three $\left(H_{3}\right)$. If we assume prior odds ratios $P\left(H_{i}\right) / P\left(H_{j}\right)=1 \forall i, j$, then the posterior odds ratio relating $H_{i}$ and $H_{j}$ is given by

$$
K_{i j}=\frac{P\left(H_{i}\right) \iiint f\left(\mathbf{m} \mid p, q, \sigma_{i}, m_{1}, H_{i}\right) f\left(p, q, \sigma_{i} \mid H_{i}\right) d \sigma_{i} d p d q}{P\left(H_{j}\right) \iiint f\left(\mathbf{m} \mid p, q, \sigma_{j}, m_{1}, H_{j}\right) f\left(p, q, \sigma_{j} \mid H_{j}\right) d \sigma_{j} d p d q} .
$$

The posterior probabilities $\pi_{i}, i=1,2,3$ are given by $\pi_{i}=1 /\left(1+\sum_{i \neq j} K_{j i}\right)$.

\section{The EM algorithm}

Recall that we have right-censored return data in our system. The EM algorithm is an appropriate method to estimate the parameters of the return process under these conditions. We first describe this algorithm (using notation from Cox and Oakes 1984) and then illustrate its application to our data. Let $T=\left(T_{1}, \ldots, T_{n}\right)$ be the iid random variables that would be observed if there was no censoring, and let $\tilde{\theta}$ denote the parameters of the distribution of $T_{i}$. Let $x_{i}$ denote the actual (possibly censored) observation for data point $i$, and let $v_{i}=1$ if the data is uncensored and 0 otherwise. Let $x=\left(x_{1}, \ldots, x_{n}\right)$ and $v=\left(v_{1}, \ldots, v_{n}\right)$.

For a given $\theta$, define $l_{0}(\theta)=l_{0}(\theta ; T)$ to be the log-likelihood of $\theta$ based on the uncensored data $\left(T_{1}, \ldots, T_{n}\right)$ and define $l(\theta)=l(\theta ; x, v)$ to be the log-likelihood of the observed data $(x, v)$. Define $Q\left(\theta^{\prime}, \theta\right)=E\left(l_{0}\left(\theta^{\prime} ; T \mid x, v ; \theta\right)\right)$ to be the conditional expectation of the log-likelihood of $\theta^{\prime}$ based on $T$, given the observations $(x, v)$. Here the expectation is taken with respect to a distribution with parameter $\theta$. Starting with an initial value $\hat{\theta}_{0}$, the two steps of the expectation maximization (EM) algorithm are: Expectation step: Calculate $Q\left(\theta^{\prime}, \hat{\theta}_{j}\right)$. (Here $\theta^{\prime}$ is a dummy variable and $\hat{\theta}_{j}$ is the current estimate of $\tilde{\theta}$.)

Maximization step: Find the value of $\theta^{\prime}$ that maximizes $Q\left(\theta^{\prime}, \hat{\theta}_{j}\right)$. This is the new estimate $\hat{\theta}_{j+1}$.

This recursive procedure yields a sequence of estimates, $\hat{\theta}_{j}, j=1, \ldots$ that converges to the maximum likelihood estimator of $\tilde{\theta}$ based on the observations.

Let us illustrate this method for our problem. The uncensored data are $s_{i}=$ sale time of item $i, i=1, \ldots n$ and $r_{i}=$ return time of item $i, i \ldots m$, where $m \leq n$. We 
set $r_{i}=\infty$ for items that will not be returned, and index the items so that units $i=$ $1 \ldots m$ have been returned. Let $T=\left(T_{1}, \ldots, T_{n}\right)$ be iid random variables representing the elapsed time from the sale to the return of the $n$ cameras. We assume that the delay for returned cameras is geometric with parameter $\tilde{q}$, and denote the return probability of a camera by $\tilde{p}$. Let $t$ be the current time. Then, $x_{i}=\min \left(r_{i}-s_{i}, t-s_{i}\right)$ and $v_{i}=I_{\left\{r_{i} \leq t\right\}}$.

The likelihood function if there was no censoring (i.e., the true values of $r_{i}, i=$ $m+1, \ldots, n$ were known) is

$$
\prod_{\left\{i \mid r_{i}<\infty\right\}} p q(1-q)^{r_{i}-s_{i}} \prod_{\left\{i \mid r_{i}=\infty\right\}}(1-p)=(1-p)^{n-k} p^{k} q^{k}(1-q)^{\sum_{\left\{i \mid r_{i}<\infty\right\}}\left(r_{i}-s_{i}\right)},
$$

where $k$ equals the number of items that eventually get recycled. The log-likelihood is given by

$$
l_{0}(p, q ; T)=k \log p+(n-k) \log (1-p)+k \log q+\sum_{\left\{i \mid r_{i}<\infty\right\}}\left(r_{i}-s_{i}\right) \log (1-q),
$$

and its conditional expectation is

$$
\begin{aligned}
Q\left(p^{\prime}, q^{\prime}, p, q\right)= & E(k \mid x, v ; p, q)\left\{\log p^{\prime}-\log \left(1-p^{\prime}\right)+\log q^{\prime}\right\}+n \log \left(1-p^{\prime}\right) \\
& +E\left(\sum_{i=1}^{n} I\left\{r_{i}<\infty\right\}\left(r_{i}-s_{i}\right) \mid x, v ; p, q\right) \log \left(1-q^{\prime}\right),
\end{aligned}
$$

where

$$
E(k \mid x, v ; p, q)=m+\sum_{i=m+1}^{n} \frac{p(1-q)^{t-s_{i}+1}}{1-p+p(1-q)^{t-s_{i}+1}}
$$

and

$E\left(\sum_{i=1}^{n} I\left\{r_{i}<\infty\right\}\left(r_{i}-s_{i}\right) \mid x, v ; p, q\right)=\sum_{i=1}^{m}\left(r_{i}-s_{i}\right)+\sum_{i=m+1}^{n}\left(t-s_{i}+\frac{1-q}{q}\right) \frac{p(1-q)^{t-s_{i}+1}}{1-p+p(1-q)^{t-s_{i}+1}}$

Setting the derivatives of $Q\left(p^{\prime}, q^{\prime}, p, q\right)$ with respect to $p^{\prime}$ and $q^{\prime}$ equal to zero and solving for $p^{\prime}$ and $q^{\prime}$ yields the following recursive relationship for the point estimates of $\tilde{p}$ and $\tilde{q}$ :

$$
\begin{gathered}
\hat{p}_{j+1}=\frac{1}{n}\left(m+\sum_{i=m+1}^{n} \frac{\hat{p}_{j}\left(1-\hat{q}_{j}\right)^{t-s_{i}+1}}{1-\hat{p}_{j}+\hat{p}_{j}\left(1-\hat{q}_{j}\right)^{t-s_{i}+1}}\right), \\
\hat{q}_{j+1}=\frac{m+\sum_{i=m+1}^{n} \frac{\hat{p}_{j}\left(1-\hat{q}_{j}\right)^{t-s_{i}+1}}{1-\hat{p}_{j}+\hat{p}_{j}\left(1-\hat{q}_{j}\right)^{t-s_{i}+1}}}{m+\sum_{i=1}^{m} r_{i}-s_{i}+\sum_{i=m+1}^{n}\left(t-s_{i}+1+\frac{1-\hat{q}_{j}}{\hat{q}_{j}}\right) \frac{\hat{p}_{j}\left(1-\hat{q}_{j}\right)^{t-s_{i}+1}}{1-\hat{p}_{j}+\hat{p}_{j}\left(1-\hat{q}_{j}\right)^{t-s_{i}+1}}} .
\end{gathered}
$$




\section{References}

[1] Baskett, F. K., M. Chandy, R. R. Muntz and F. G. Palacios, "Open, Closed and Mixed Networks of Queues with Different Classes of Customers," J. Assoc. Computing Machinery, 22 (1975), 248-260.

[2] Cox, D. R. and D. Oakes, Analysis of Survival Data, Chapman and Hall, London, 1984.

[3] Dempster, A. P., N. M. Laird and D. B. Rubin, "Maximum Likelihood from Incomplete Data via the EM Algorithm (with Discussion)," J. Royal Stat. Soc. Series B, 339 (1977), 1-22.

[4] Dhrymes, P.J., Distributed Lags. North-Holland, Amsterdam, 1985.

[5] Goldstein, L., "The Strategic Management of Environmental Issues: A Case Study of Kodak's Single-use Cameras," M.S. Thesis, Sloan School of Management, MIT, Cambridge, MA, 1994.

[6] Kelle, P. and E. A. Silver, "Forecasting the Returns of Reusable Containers," Journal of Operations Management, 8 (1989a), 17-35.

[7] Kelle, P. and E. A. Silver, "Purchasing Policy of New Containers Considering the Random Returns of Previously Issued Containers," IIE Transactions, 21 (1989b), 349-354.

[8] Kelly, F.P., Reversibility and Stochastic Networks. Wiley, New York, 1979.

[9] Lariviere, M. A. and E. L. Porteus, "Stalking Information: Bayesian Inventory Management with Unobserved Lost Sales," Research Paper \# 1367, Graduate School of Business, Stanford University, Stanford, CA, 1995.

[10] Nahmias, S., "Demand Estimation in Lost Sales Inventory Systems," Working Paper, Santa Clara University, Santa Clara, CA, 1994. 
[11] Rubio, R. and L. M. Wein, "Setting Base Stock Levels Using Product-form Queueing Networks," Management Science 42 (1996), 259-268.

[12] Solberg, J. J., "A Mathematical Model of Computerized Manufacturing Systems," in Proceedings of the 4th International Conference on Production Res., Tokyo, Japan, 1977, 22-30.

[13] Spearman, M. L., D. L. Woodruff, and W. J. Hopp, "CONWIP: A Pull Alternative to Kanban," International J. Production Res., 28 (1990), 879-894.

[14] Toktay, L. B., "An Analysis of Two Supply Chain Management Problems," Ph.D. Thesis, Operations Research Center, MIT, Cambridge, MA (1998), in preparation.

[15] Thierry, M.C., M. Salomon, J. A. E. E. van Nunen, and L.N. van Wassenhove, "Strategic Production and Operations Management Issues in Product Recovery Management," California Management Review, 37 (1995), 114-135.

[16] van der Laan, E. A., M. Salomon, and R. Dekker, "Production Planning and Inventory Control for Remanufacturable Durable Products," Econometric Institute Report Series \#9531-A, Erasmus University, Rotterdam, The Netherlands, 1995 .

[17] Whitt, W., "Open and Closed Models for Networks of Queues," ATGT Bell Laboratories Technical Journal, 63 (1984), 1911-1979.

[18] Whitt, W., "Towards Better Multi-class Parametric-decomposition Approximations for Open Queueing Networks," Annals of Operations Research, 48 (1994), 221-248.

[19] Zellner, A., An Introduction to Bayesian Inference in Econometrics. Malabar, Florida: Robert E. Krieger Publishing Company, 1987. 